\title{
The FBXW7 tumor suppressor inhibits breast cancer proliferation and promotes apoptosis by targeting MTDH for degradation
}

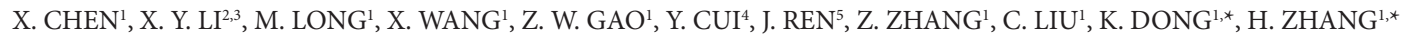 \\ ${ }^{1}$ Department of Medical Laboratory and Research Center; Tangdu Hospital; Fourth Military Medical University; Xian, China; ${ }^{2}$ Department \\ of Ophthalmology; Xi 'an No. 4 Hospital; Xi' an, China; ${ }^{3}$ Department of Ophthalmology, Eye Institute of Chinese PLA, Xijing Hospital, Fourth \\ Military Medical University, Xi'an, China; ${ }^{4}$ Department of Blood Transfusion, Tangdu Hospital, The Fourth Military Medical University, Xian, \\ China; ${ }^{5}$ Department of Medical, AstraZeneca China
}

*Correspondence:td_zhz@163.com,tddk01@126.com

Received February 28, 2017/ Accepted June 9, 2017

\begin{abstract}
Metadherin $(\mathrm{MTDH})$ is an oncoprotein and is expressed at high levels in a wide variety of human carcinomas, which represents an important genetic determinant and regulates multiple events in tumorigenesis. MTDH promotes breast cancer cell proliferation and tumorigenesis through the activation of numerous signaling pathways. Currently, the mechanism regulating MTDH expression is poorly understood. Here we identified that FBXW7, a component of E3 ubiquitin ligase, targets MTDH for ubiquitin-mediated degradation. Forced overexpression of FBXW7 could decrease the level of MTDH protein, and inhibition of endogenous FBXW7 expression remarkably increases the MTDH protein abundance. More importantly, overexpression of FBXW7 could lead to proliferation arrest and apoptosis in breast cancer cells through targeting MTDH degradation. These data suggest that FBXW7, a tumor suppressor, inhibits breast cancer cell proliferation and promotes apoptosis at least partially through targeting MTDH for proteolysis. This new regulatory mechanism of MTDH by FBXW7 represents a new pathway for malignant phenotype turnover in human breast cancer.
\end{abstract}

Key words: MTDH, FBXW7, Ubiquitin-proteasome degradation, cell proliferation, cell apoptosis, breast cancer

Metadherin (MTDH), also known as AEG-1, LYRIC or 3D3, is a transmembrane protein containing 582 amino acids [1]. MTDH is an oncoprotein and is expressed at high levels in a wide variety of human carcinomas, including glioma, melanoma and neuroblastoma, as well as carcinomas of the breast, prostate, liver and esophagus [2-4]. Studies have shown that MTDH plays a crucial role in regulation of the breast cancer development, progression and metastasis, and it is a prognostic factor in breast cancer [5]. Statistical analyses have shown a significant correlation of MTDH expression with the clinical stages and prognosis of the patients. In normal human breast tissues, the expression of MTDH is decreased or is completely absent, while it is widely overexpressed in nearly all of breast tumor tissues and tumor cell lines examined [1, 6-8]. In addition, inhibition of MTDH expression in breast cancer cells can interfere with the proliferation of these cells [9], indicating its potential as a specific new target in clinical-targeted therapeutics of breast cancer.

In these studies, we wanted to find the proteins capable of interacting with MTDH and FBXW7 was identified as a candidate of such proteins. FBXW7 (F-box and WD repeat domain-containing 7, also known as FBW7, cdc4) is one of the F-box proteins that function as substrate for the $\mathrm{SCF}^{\mathrm{Fbxw}}$ (a complex of Skp1, CUL1 and F-box proteins) E3 ubiquitin ligase. The ubiquitin proteasome system regulates the turnover of a number of proteins and plays an essential role in maintaining normal cellular function [10]. Dysregulation of ubiquitin-mediated proteolysis results in the development of a variety of human cancers [11]. FBXW7, as an E3 ligase, plays an important role in mediating the ubiquitination and subsequent proteolytic turnover of protein substrates [12]. Our previous studies have shown that FBXW7 is a tumor suppressor and is inactivated in numerous human malignances, including breast cancer, by gene mutation and expression downregulation $[13,14]$. The FBXW7 gene encodes three protein isoforms with distinct subcellular localization, as the FBXW7 $\alpha$ (nuclear) FBXW7 $\beta$ (cytoplasmic) and FBXW7 $\gamma$ (nucleolar) [15]. FBXW7 is also known to regulate the degradation of several oncoproteins, such as MYC [16, 17], cyclin E [15, 18], Notch [19], KLF-5 [20], and PGC1a $[21,22]$, many of its substrates have yet to be identified.

We found that FBXW7 could interact with MTDH in breast cancer cells MDA-MB-231. However, whether and how FBXW7 affects MTDH protein levels and further influ- 
ences the MTDH functional roles within breast cancer cells has not been cleared so far. In this article, we found that FBXW7 negatively regulated MTDH protein level via the ubiquitination pathway. Depletion of endogenous FBXW7 stabilized MTDH protein, whereas overexpression of FBXW7 promoted degradation of MTDH and further impaired cell proliferation and apoptosis. In sum, our results showed that FBXW7, as an ubiquitin ligase of MTDH, was a critical negative regulator of MTDH protein abundance.

\section{Materials and methods}

Antibodies and chemicals. Antibodies against FBXW7 (ab171961), Flag tags (ab122902), Ubiquitin (ab7780), were purchased from Abcam (UK). The antibody against MTDH (13860-1-AP) was obtained from Proteintech (USA). The antibodies against His (sc-804), GST (sc-459) as well as all secondary antibodies were from Santa Cruz Biotechnology (CA, USA). Cycloheximide (CAS Number: 66-81-9) and MG132 (CAS Number: 133407-82-6) were purchased from Sigma (MO, USA). Cycloheximide was dissolved in water and the concentration for cell treatment was $25 \mu \mathrm{M}$ at indicated time points. MG132 was dissolved in DMSO and dosage was $10 \mu \mathrm{M}$.

Cell cultures and transfection. Human breast cancer cell lines MDA-MB-23, SKBR3 and human embryonic cells HEK293 were purchased from ATCC (American Type Culture Collection) and cultured in Dulbecco's modified Eagle's medium supplemented with $10 \%$ fetal bovine serum and $2 \mathrm{mM} \mathrm{L}$-glutamine. All cells were incubated at $37^{\circ} \mathrm{C}$ with $5 \% \mathrm{CO}_{2}$.

The cells were seeded in 6 -well plates at $2.5 \times 10^{4}$ cells per well and grown overnight to $80 \%$ confluence prior to transfection. All transfections for plasmid and shRNA were performed with Lipofectamine 2000 (Invitrogen, Carlsbad,
CA, USA) according to the manufacturer's instructions. To select neomycin-resistant cells, $500 \mu \mathrm{g}$ G418 (Invitrogen, Carlsbad, CA, USA) was applied.

Plasmid construction. The pCDNA3.1-MTDH and pCDNA3.1-FBXW7a recombination vectors were constructed by inserting the full-length MTDH and FBXW7 $a$ cDNAs (generated by RT-PCR from total mRNA of MDA-MB-231 cells) into pCDNA3.1(+) vectors (Invitrogen), respectively [16]. The prokaryotic expression vectors for pGSTag-FBXW7a were constructed by cloning the FBXW7a cDNAs into pGSTag vectors (Amersham Biosciences). The MTDH, FBXW7 $\alpha$, FBXW7 $\beta$ and FBXW7 $\gamma$ cDNAs were inserted into pFlag-CMV vectors (Invitrogen) to construct the pFlag-CMV-MTDH, pFlag-CMV-FBXW7 $a$, pFlag-CMV-FBXW7 $\beta$ and Flag-CMV-FBXW7 $\gamma$ recombination vectors, respectively. The primer sequences used in this part of experiments are shown in Table 1.

DNA template encoding FBXW7 shRNA was designed and synthesized (Genepharma Inc, Suzhou, China) as follows: the 21-nt target sequence (FBXW7, NM_033632, 1661-1681 bp) was chosen as a sense strand followed by a 9-nt spacer and a complementary antisense strand sequence as shown in Table 1 [23]. The shRNA was annealed and subcloned into pSilencer4.1-CMV neo (Amibion, Austin, TX, USA) BamHI and HindIII enzyme sites downstream of CMV promoter, and recombinant vector was named as pSilencer4.1-siFBXW7. The shRNA designed towards a non-specific (NS) sequence was also used as a negative control and named as pSilencer4.1-siNC. BLAST search against EST libraries was performed to confirm that no other human gene was targeted.

Co-immunoprecipitation. Co-immunoprecipitation assay was performed as previously described [24]. In brief, $48 \mathrm{~h}$ after transfection, the cells were washed with PBS and lysed in modified RIPA buffer (20 mM Tris (pH 7.5), $150 \mathrm{mM} \mathrm{Nacl,}$

Table 1. The primer sequences used in this part of experiment.

\begin{tabular}{|c|c|}
\hline vector & primer \\
\hline \multirow{2}{*}{ pcDNA3.1-MTDH } & Forward:5'-CCGAATTCATGGCTGCACGGAGC-3' \\
\hline & Reverse: 5'-CGTCTAGATCACGTTTCTCGTCTG-3' \\
\hline \multirow{2}{*}{ pcDNA3.1-FBXW7a } & Forward:5'-CCGAAGCTTATGAATCAGGAACTGCTCTCTG-3 \\
\hline & Reverse:5'-CGTCTAGATCACTTCATGTCCACATCAAAGTCC-3 \\
\hline \multirow{2}{*}{ pFlag-CMV-MTDH } & Forward:5'-CCGAATTCATGGCTGCACGGAGC-3' \\
\hline & Reverse: 5'-CGTCTAGATCACGTTTCTCGTCTG-3' \\
\hline \multirow{2}{*}{ pFlag-CMV-FBXW7a } & Forward:5'-CCGAAGCTTATGAATCAGGAACTGCTCTCTG-3 \\
\hline & Reverse:5'-CGTCTAGATCACTTCATGTCCACATCAAAGTCC-3 \\
\hline \multirow{2}{*}{ pFlag-CMV-FBXW7 $\beta$} & Forward:5'-CCGAAGCTTATGAATCAGGAACTGCTCTCTG-3 \\
\hline & Reverse:5'-CGTCTAGATCACTTCATGTCCACATCAAAGTCC-3 \\
\hline \multirow{2}{*}{ pFlag-CMV-FBXW7 $\gamma$} & Forward:5'-CCGAAGCTTATGTCAAAACCGGGAAAACCTAC-3 \\
\hline & Reverse:5'-CGTCTAGATCACTTCATGTCCACATCAAAGTCC-3 \\
\hline \multirow{2}{*}{ pGSTag-MTDH } & Forward:5'-CCGAATTCTAATGGCTGCACGGAGC-3' \\
\hline & Reverse: 5'-CGCTCGAGTCACGTTTCTCGTCTG-3' \\
\hline \multirow{2}{*}{ pSilencer4.1-ShFBXW7 } & Sense:5-GATCCCCGCTGTGTTCAATATGATTTCAAGAGAATCATATTGAACACAGCGGACA-3’ \\
\hline & Antisense:5-AGCTTGTCCGCTGTGTTCAATATGATTCTCTTGAAATCATATTGAACACAGCGGG-3' \\
\hline
\end{tabular}


$0.5 \%$ Triton X-100 and $1 \mathrm{mM}$ protease inhibitor PMAF) in the cold. The lysates were incubated with antibodies $(5 \mu \mathrm{g} /$ sample) overnight at $4{ }^{\circ} \mathrm{C}$ with gentle agitation. The next day, protein A/G Plus Agarose (Santa Cruz, CA, USA) was added to the mixture of lysates and antibodies ( $20 \mu \mathrm{l} /$ tube), followed by a $1 \mathrm{~h}$ incubation in the cold. The samples were washed and boiled for $5 \mathrm{~min}$ at $95^{\circ} \mathrm{C}$ in $60 \mu \mathrm{l} 2 \times \mathrm{SDS}$ loading buffer. Then the beads were collected by brief centrifugation and the supernatant fraction was assessed by SDS-PAGE.

Protein identification by mass spectrometry. The analysis of proteins by liquid chromatography combined with random mass spectrometry (LC/MS/MS) was performed as previously described [24], and it was conducted by Beijing Proteome Research Center, China.

Protein purification and GST-pulldown. The prokaryotic recombination expression vectors of GST-FBXW7 $\alpha$ and GST were expressed in BL21 (DE3) pLysS Escherichia coli, respectively. To obtain the expressed proteins, the cell lysates were prepared following manufacturer's protocol (GE Healthcare Bio-Sciences $A B$ ) and were allowed to bind to Glutathione Sepharose $4 \mathrm{~B}$ for $30 \mathrm{~min}$ at room temperature, washed 3 times with buffer-A $\left(2.7 \mathrm{mM} \mathrm{KCl}, 140 \mathrm{mM} \mathrm{NaCl}, 10 \mathrm{mM} \mathrm{Na}_{2} \mathrm{HPO}_{4}\right.$, $1.8 \mathrm{mM} \mathrm{KH}_{2} \mathrm{PO}_{4}, \mathrm{pH} 7.3$ ), and then eluted with $600 \mu \mathrm{l}$ of buffer-B (50 mM Tris- $\mathrm{HCl}, 10 \mathrm{mM}$ reduced glutathione, $\mathrm{pH}$ 8.0). The eluted proteins GST-FBXW7 $\alpha$ and GST were analyzed by SDS-PAGE and stained with Coomassie Blue to check for the protein purity [27]. Then, $50 \mu \mathrm{g}$ of GST or GST-MTDH were incubated with $50 \mu \mathrm{l}$ (50\% slurry) of Glutathione HiCap Matrix (Qiagen) in buffer-C (50 mM Tris- $\mathrm{HCl}$, $200 \mathrm{mM} \mathrm{NaCl}, 1 \mathrm{mM}$ EDTA, 1\% NP40, 1 mM DTT, $10 \mathrm{mM}$ $\mathrm{MgCl}_{2}, \mathrm{pH} 8.0$ and protease inhibitor PMSF) at room temperature with rocking. $2 \mathrm{~h}$ later, the mixtures were collected in separate tubes. $500 \mu \mathrm{g}$ of MDA-MB-231 cell lysate, transfected with pFlag-CMV-MTDH, was added to each tube, containing Glutathione HiCap Matrix treated GST or GSTGST-FBXW7 $\alpha$, and kept overnight under same conditions. The next day, beads were washed 3 times with buffer-A and eluted with $50 \mu \mathrm{l}$ of buffer-D (50 mM Tris- $\mathrm{HCl}$ pH 8.0, $400 \mathrm{mM}$ $\mathrm{NaCl}, 50 \mathrm{mM}$ reduced glutathione, $1 \mathrm{mM}$ EDTA, $1 \mathrm{mM}$ DTT) and then subjected to western blotting analysis [28].

Ubiquitin ligase activity assay. In vivo ubiquitination assay was performed as described previously [21]. Briefly, MAD-MB-231cells were transfected with the indicated plasmids. $48 \mathrm{~h}$ post transfection, $10 \mu \mathrm{M}$ MG132 was added to the cell for $4 \mathrm{~h}$. Then the cells were lysed with modified RIPA buffer and subjected to co-immunoprecipitation with anti-MTDH antibody. Immunocomplexes were separated on $12 \%$ gradient gels and immunoblotting was performed using Ub antibody.

Cell proliferation assay. Cell proliferation was estimated by MTT (Sigma, St. Louis, MO, 197 USA) assay at indicated time point as described before [24]. All experiments were performed in triplicate and absorbance was determined on a microreader (Bio-Rad) at $570 \mathrm{~nm}$. The cell proliferation diagram was plotted using the absorbance at each time point.
Cell apoptosis analysis. Cell apoptosis was detected using the annexin V-FITC apoptosis detection kit (KeyGEN BioTECH, Nanjing, China) with flow cytometer (BD Biosciences, San Jose, USA). Briefly [25], cells were pelleted, suspended in Annexin V-fluorescein isothiocyanate $(0.5 \mathrm{mg} / \mathrm{ml})$ and propidium iodide $(0.6 \mathrm{mg} / \mathrm{ml})$. Following that, cells were examined using FACS calibur flow cytometer and then analyzed with WinMDI software (The Scripps Institute, La Jolla, CA, USA).

Data analysis and statistics. Each experiment was repeated 3 times and all results were expressed as mean \pm standard deviation $( \pm \mathrm{s})$. All data were performed using SPSS version 17.0. Student's t-test and one-way ANOVA followed by Dunnett's multiple comparison tests were assessed. Values of $\mathrm{p}<0.05$ were considered as significant and were indicated by asterisks in the figures.

\section{Results}

Identification of FBXW7 as a partner of MTDH protein in breast cancer cells. To identify the natural protein partners of MTDH in breast cancer cells, Flag$\mathrm{MTDH}$ protein was expressed in human breast cancer cell line MDA-MB-231. We analyzed MTDH interactive proteins by LC/MS/MS after sequential Flag immunoprecipitation and peptide elution [24], and discovered that MTDH complexes contained FBXW7 (Figure 1A). FBXW7 (F-box and WD repeat domain-containing 7, also known as SEL-10, hAgo, hCdc4) is a substrate recognition component of the SCF E3 ubiquitin-protein ligase complex which mediates ubiquitination and subsequent proteasomal degradation of target proteins [26]. To further reveal the endogenous interaction between FBXW7 and MTDH, we performed co-immunoprecipitation assays in MDA-MB231 cells and found that there was an integration band of FBXW7 and MTDH (Figure 1B, 1C). To see if FBXW7 has a similar interaction with MTDH in vitro, we used purified GST-MTDH in a pull-down assay and found that FBXW7 specifically interacted with MTDH (Figure 1D). Collectively, it could be concluded that FBXW7 had an interaction with MTDH both in vivo and in vitro.

FBXW7 negatively regulates MTDH protein stability. FBXW7 family has three isoforms: FBXW7 $\alpha$, FBXW7 $\beta$ and FBXW7 $\gamma$ [15]. To see if all three of the isoforms have a similar impact on $\mathrm{MTDH}$, we examined the expression of $\mathrm{MTDH}$ in MDA-MB-231 cells in the presence of forced overexpression of each FBXW7 isoform. Studies showed that all the three isoforms have similar impact on MTDH. Indeed, overexpression of Flag-CMV-FBXW7 $\alpha, \beta$ and $\gamma$ could all reduce the MTDH abundance (Figure 2A). Because FBXW7a is the highest expressed and stable isoform of FBXW7 in breast cancer cell lines $[27,28]$, Flag-FBXW7a was transfected with increasing vector amounts (1.0, 2.0 and $3.0 \mu \mathrm{g}$ ) into MDA-MB-231 cells, and the result showed an inverse abundance of MTDH protein levels (Figure 2B). The data 
Figure1. FBXW7 was a partner of MTDH protein. A) FBXW7 was identified as one of MTDH complexes from human breast cancer cell line MDA-MB-231. Affinity purified Flag-MTDH complexes were silver stained from MDA-MB-231 cells. Specific MTDH-interacting proteins were identified by mass spectrometry and were indicated. B) Proteins were immunoprecipitated from MDA-MB-231 cell lysates with antibodies against MTDH and checked for FBXW7. Western blotting results showed positive bands in both anti-MTDH and anti-FBXW7 antibodies panels. C) Proteins were immunoprecipitated from MDA-MB-231 cell lysates with antibodies against FBXW7 and checked for MTDH. Western blotting results showed positive bands in both anti-MTDH and antiFBXW7 antibodies panels. D) GST and GST-MTDH (500 $\mu \mathrm{g})$ were used for GST pull down assay with MDA-MB-231 cell lysates, transfected with pcDNA3.1-MTDH-Flag vector, and western blotting results showed positive bands in all three different primary antibodies of anti-MTDH, antiFBXW7, and anti-GST.
A
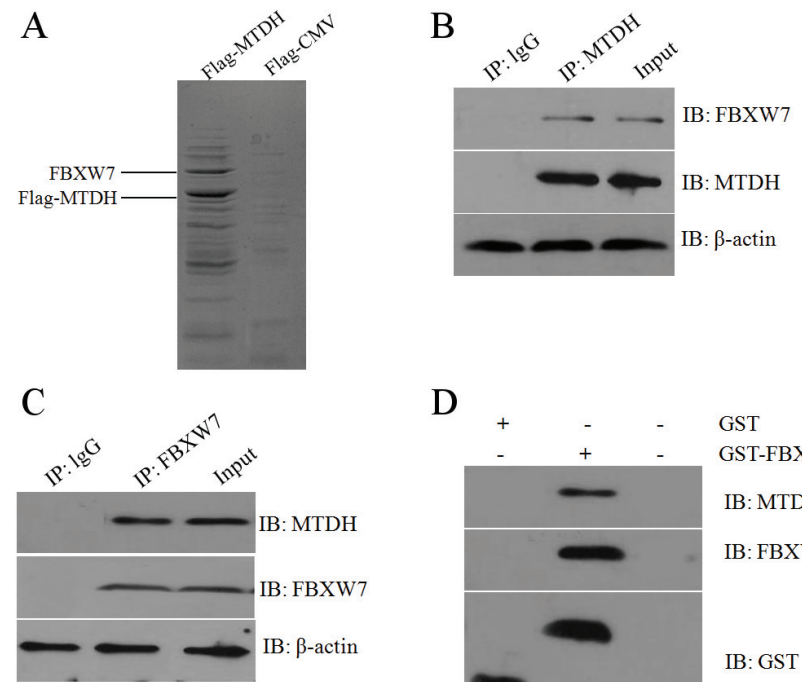

$\mathrm{D}$

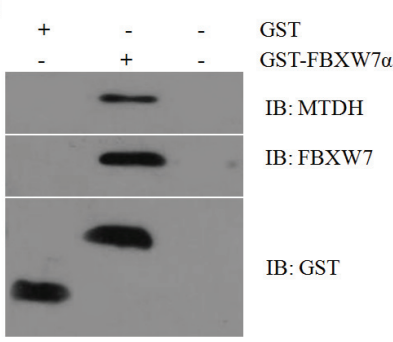


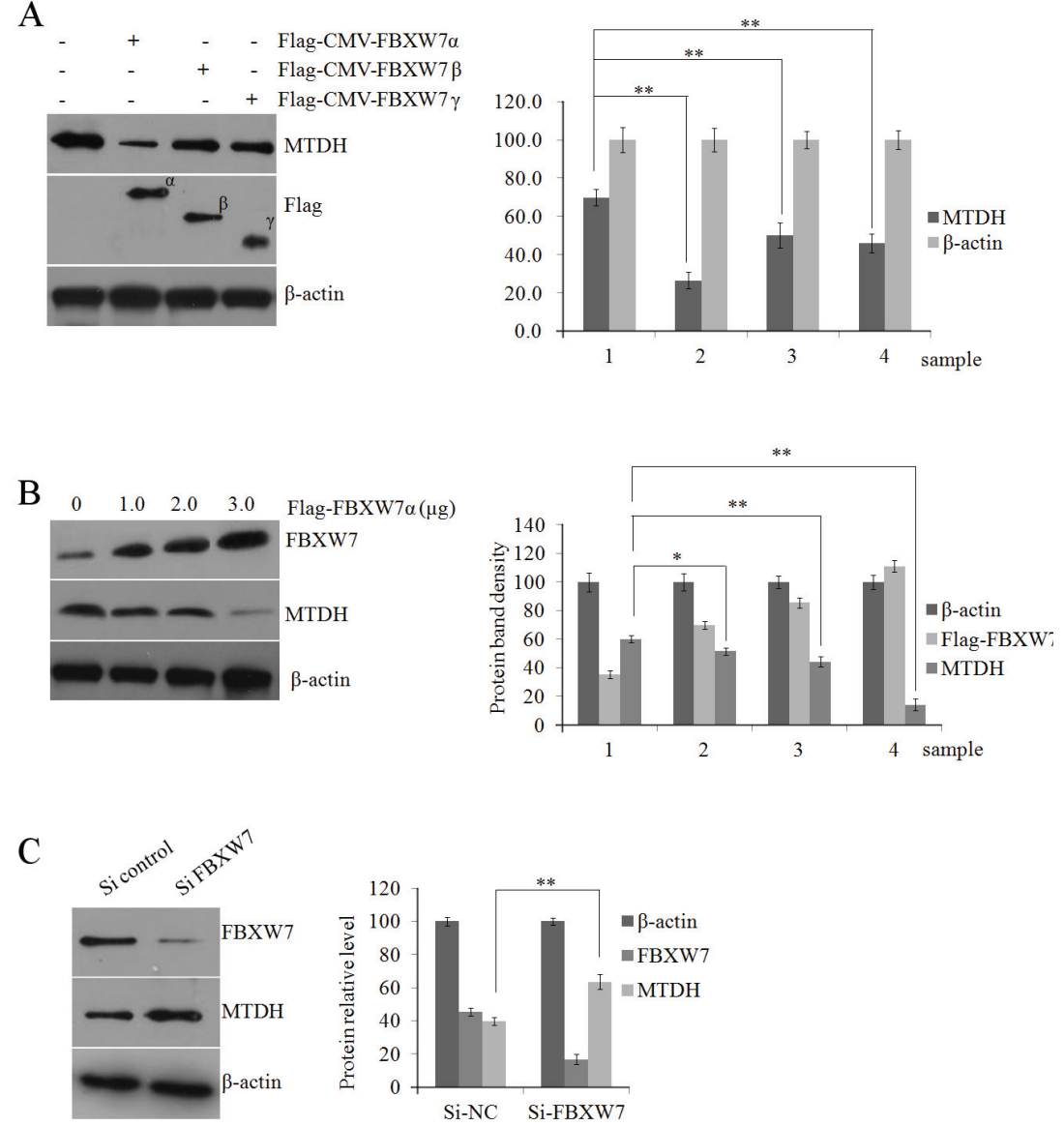

Figure 2. FBXW7 negatively regulated MTDH protein stability. A) Left figure, MDA-MB-231 cells were transfected with different combinations of expression plasmids (Flag-CMV, Flag-CMV-FBXW7 $\alpha, \beta$ and $\gamma$ ) and the MTDH protein levels were all decreased compared to control group, especially in the MDA-MB-231 cells transfected with Flag-CMV-FBXW7 $\alpha$. Right figure, densitometry of the immunoblots shown in $B$ left figure $\left({ }^{*} p<0.05\right.$, ${ }^{* *} p<0.01$ vs control, $n=3$ ). B) Left figure, MDA-MB-231 cells were transfected with 1.0, 2.0 and $3.0 \mu \mathrm{g}$ Flag-CMV-FBXW7 $\alpha$ respectively, western blotting showed an inverse expression of MTDH. Right figure, densitometry of the immunoblots shown in B left figure $\left({ }^{*} p<0.05,{ }^{* *} p<0.01\right.$ vs control, $\left.n=3\right)$. C) Left figure, knock-down of FBXW7 with shRNA increases the MTDH protein levels in HEK293 cells compared to control cells. Right figure, densitometry of the immunoblots shown in C left figure $\left({ }^{*} \mathrm{p}<0.05,{ }^{* *} \mathrm{p}<0.01\right.$ vs control, $\left.\mathrm{n}=3\right)$. 
demonstrated that FBXW7 overexpression led to decreasing abundance of MTDH protein level. To further study the role of FBXW7 in the regulation of MTDH stability under endogenous conditions, we knocked down the FBXW7 expression in HEK293 cells by shRNA. Western blotting analysis revealed that depletion of endogenous FBXW7 expression elevated MTDH abundance when compared with control cells (Figure 2C). These results suggest that FBXW7 is a negative regulator of MTDH.

FBWX7 regulates MTDH protein stability through ubiquitination. Since FBXW7, an E3 ubiquitin ligase, negatively regulates MTDH stability, we thought it was a matter of interest to check whether FBXW7 played an E3 ubiquitin ligase role for MTDH degradation. To confirm this possibility, we overexpressed MTDH together with or without Flag-FBXW7a in MDA-MB-231 cells. Whole cell lysates were treated with proteasome inhibitor MG132 $48 \mathrm{~h}$ after transfection, and then they were immunoprecipitated using anti-MTDH antibody. Western blotting results showed that the level of poly-ubiquitination MTDH was markedly increased in the presence of FBXW7 compared to control groups (Figure 3A). To certify the role of FBXW7 in mediating MTDH ubiquitination under physiological conditions, we knocked down the FBXW7 gene expression in HEK293 cells by shRNA, and then conducted the immunoprecipitation assay with anti-MTDH antibody using the HEK293 cell lysates which were pre-treated with the proteasome inhibitor MG132. Western blotting analysis showed that deletion of endogenous FBXW7 significantly reduced the MTDH ubiquitination level compared to control groups (Figure 3B). Altogether, these results demonstrate that FBXW7 could ubiquitinate the MTDH.

FBXW7 promotes proteasome-mediated degradation of MTDH. We have shown that FBXW7 negatively regulated the steady state level of MTDH protein. To confirm the involvement of the proteasome system in FBXW7 mediated degradation of MTDH protein, we treated MDA-MB-231 cells with MG132, which stabilized MTDH and eliminated the reduction of MTDH protein level even in the presence of FBXW7 $\alpha$ (Figure 4A). To further study this function, we observed endogenous level of MTDH protein under the condition of overexpression of FBXW7a in MDA-MB-231 cells followed by treatment with $\mathrm{CHX}$ (cycloheximide, protein synthesis inhibitor). The results indicated that MTDH half-life was decreased in the condition of overexpression FBXW7a compared to control groups. The half-life of MTDH was $\sim 12 \mathrm{~h}$ in wild type cells, but it decreased to $\sim 6 \mathrm{~h}$ in the condition of overexpression FBXW7a cells (Figure 4B). Further, we also knocked down FBXW7 by shRNA in HEK293 cells and assayed the endogenous level of MTDH protein with $\mathrm{CHX}$. The half-life of MTDH protein was increased in the condition of knock-down of FBXW7 (Figure 4C). All these results confirmed that FBXW7 negatively regulates MTDH stability by targeting it for ubiquitination and degradation through ubiquitin proteasome pathway.
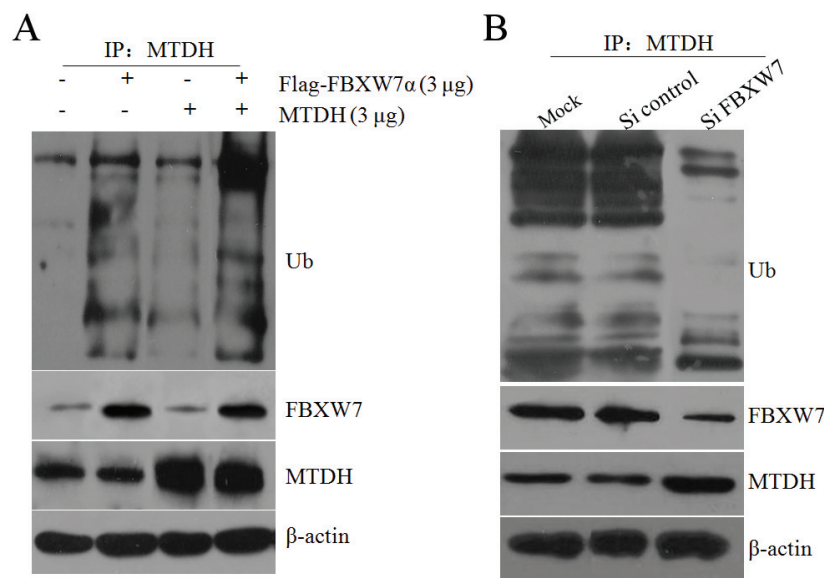

Figure 3. FBWX7 regulated MTDH protein stability through ubiquitination. A) MDA-MB-231 cells were transfected with MTDH in combination with or without FBXW7 as shown in the figure, followed by a treatment with MG132, then immunoprecipitation was performed with anti-MTDH antibody, and antibodies against Ub, MTDH and FBXW7 were used in western blotting, respectively. The results showed that level of poly-ubiquitination MTDH was markedly increased in the presence of FBXW7 compared with control groups (the fourth lane from left). B) Knock-down of FBXW7 with shRNA in HEK293 cells, followed by a treatment with MG132, then immunoprecipitation was performed with anti-MTDH antibody. Western blotting analysis showed that deletion of endogenous FBXW7 significantly reduced the MTDH ubiquitination level (the third lane from left).

FBXW7 suppresses breast cancer cell proliferation and promotes cell apoptosis through promoting MTDH degradation. We investigated the effects of overexpression of FBXW7 on cell proliferation in breast cancer cells. Cell proliferation was inhibited both in MDA-MB-231/FBXW7 $7^{+}$ and SKBR3/FBXW7 $7^{+}$cells, and the inhibitory rate was $31.7 \pm 3.21 \%$ and $30.1 \pm 3.46 \%$ compared with mock groups, respectively on $72 \mathrm{~h}$ after transfection $(\mathrm{p}<0.05)$ (Figure $5 \mathrm{~A}$, 5B). Since FBXW7 also targets several other oncoproteins for degradation [29], it was important to illuminate that FBXW7 suppressed breast cancer cell proliferation through MTDH. When the MDA-MB-231/FBXW7 ${ }^{+}$and SKBR3/FBXW7+ cells were transfected with pCDNA3.1-MTD, the inhibitory rates were lower compared to MDA-MB-231/FBXW7 $7^{+}$and SKBR3/FBXW7 $7^{+}$cells respectively on $72 \mathrm{~h}$ after transfection (Figure 5A, 5B). The cell proliferation of MDA-MB231/FBXW7 ${ }^{+}$and SKBR3/FBXW7 ${ }^{+}$were reversed by forced expression of MTDH. These results show that FBXW7 suppresses breast cancer cells proliferation by targeting MTDH degradation.

Cell apoptosis was an important cause of viability suppression, so we also performed a cell apoptosis assay with a flow cytometer. The increased rate of apoptosis in MDA-MB-231/ $\mathrm{FBXW}^{+}$and MDA-MB-231/FBXW7 ${ }^{+}+\mathrm{MTDH}$ cells was $15.38 \pm 1.63$ times and $8.89 \pm 0.89$ times compared to control cells. The increased rate of apoptosis in SKBR3/FBXW7 ${ }^{+}$ and SKBR3/FBXW7 ${ }^{+}+\mathrm{MTDH}$ cells was $6.29 \pm 0.96$ times and 
A
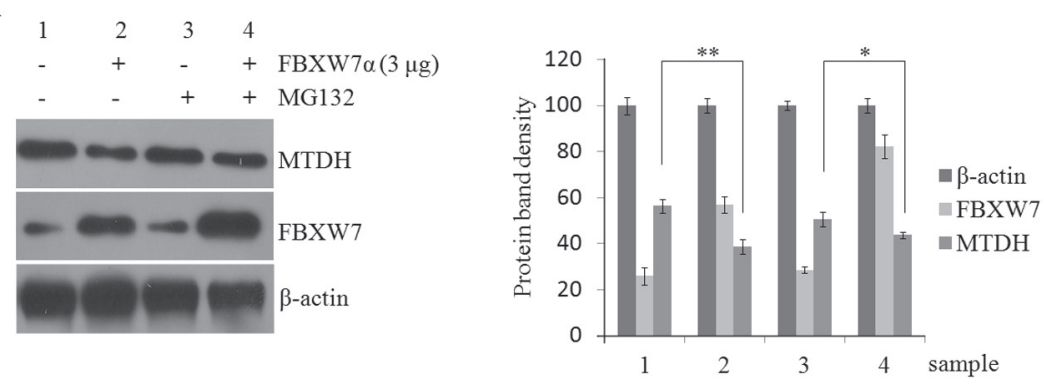

B


$\mathrm{C}$
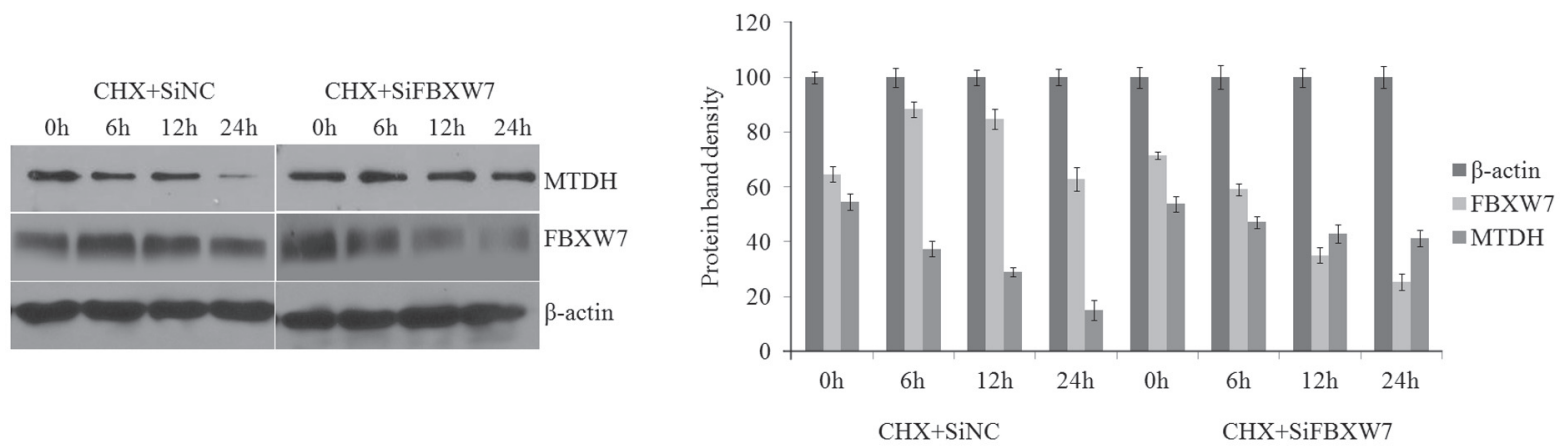

Figure 4. FBXW7 promoted proteasome-mediated degradation of MTDH. A) Left figure, MDA-MB-231 cells were transfected with plasmids expressing FBXW7 or the empty vectors, and then treated with or without MG132. The cell lysates were then checked for MTDH protein level. The results showed that with the MG132 treatment, the reduction of MTDH protein level was resected even in the condition of increased FBXW7 expression compared to MG132 untreated cells. Right figure, densitometry of the immunoblots shown in A left figure $\left({ }^{\star} p<0.05\right.$, ${ }^{* *} p<0.01 \mathrm{vs}$ control, $\left.n=3\right)$. B) Left figure, MDA-MB-231 cells were transfected with plasmids expressing FBXW7 or the empty vectors, respectively, followed by a treatment with $50 \mu M$ cycloheximide (CHX) for a time period as indicated in the figure. The lysates were then checked for MTDH level to determine the protein half-life. The half-life of MTDH protein was about $12 \mathrm{~h}$ in natural conditions, but it was reduced to $\sim 6 \mathrm{~h}$ in the conditions of FBXW7 overexpression. Right figure, densitometry of the immunoblots shown in B left figure. C) Left figure, knock-down of FBXW7 with shRNA in HEK293 cells, followed by a treatment with $50 \mu \mathrm{M}$ cycloheximide (CHX) for a time period as indicated in the figure. The lysates were then checked for MTDH level. The results showed that the MTDH protein level did not change obviously in the condition of decreased FBXW7 protein expression compared to control group. Right figure, densitometry of the immunoblots shown in C left figure. Right figure, densitometry of the immunoblots shown in C left figure.

$3.20 \pm 0.54$ times compared to control cells. FBXW7 overexpression induced cell apoptosis in MDA-MB-231 cells and SKBR3 cells. And the apoptosis of MDA-MB-231/FBXW7+ and SKBR3/FBXW7 ${ }^{+}$cells was reversed by forced expression of MTDH (Figure 5C, 5D). These results demonstrate that endogenous FBXW7 promotes cell apoptosis through targeting the endogenous MTDH for degradation. In sum, FBXW7 suppresses breast cancer cell progression through mediating MTDH degradation in some measure.

\section{Discussion}

Here, we provide several lines of evidence to support that oncoprotein MTDH is targeted by E3 ubiquitin ligase FBXW7 for proteasome degradation. First, MTDH binds to FBXW7. Second, FBXW7 negatively regulates MTDH protein stability. Third, FBWX7 regulates MTDH protein stability through ubiquitination. Fourth, FBXW7 promotes proteasome-mediated degradation of $\mathrm{MTDH}$. Addition- 
A

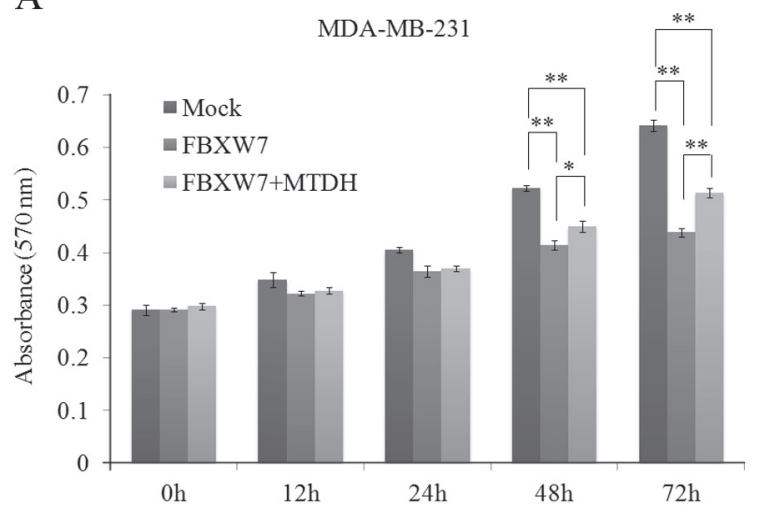

B

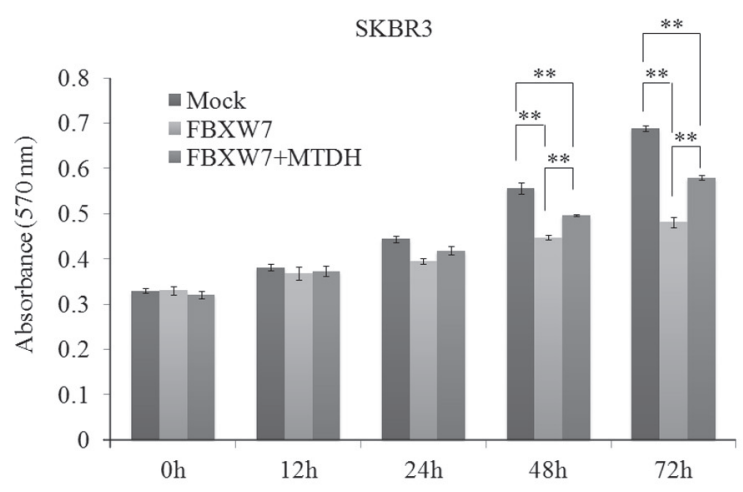

$\mathrm{C}$

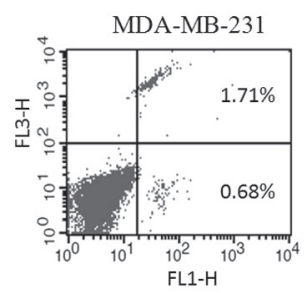

$\mathrm{D}$




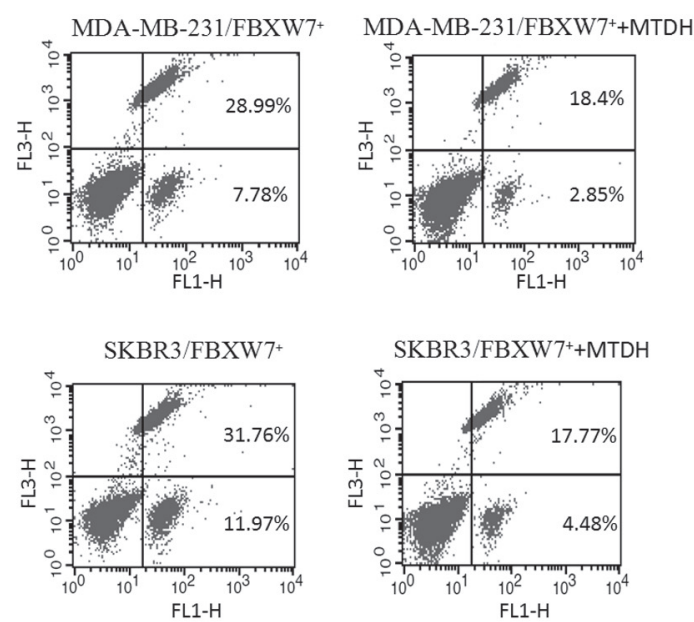

SKBR3/FBXW7++MTDH

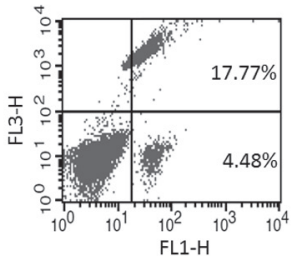

Figure5. FBXW7 suppressed breast cancer cell proliferation and promoted cell apoptosis through promoting MTDH degradation. A) and B) MDAMB-231 and SKBR3 cells transfected with the empty vectors, or with the plasmid expressing FBXW7, or co-transfected with the plasmids expressing the FBXW7 and MTDH. Cell viability was evaluated by MTT assay for a time period as indicated in the figure. The inhibitory rate $72 \mathrm{~h}$ after transfection was $31.7 \pm 3.21 \%$ and $20 \pm 2.85 \%$ in MDA-MB-231/FBXW7 $7^{+}$and MDA-MB-231/FBXW7 $7^{+}$MTDH cells compared with control cells, respectively. The inhibitory rate $72 \mathrm{~h}$ after transfection was $30.1 \pm 3.46 \%$ and $15.8 \pm 2.53 \%$ in SKBR3/FBXW7 $7^{+}$and SKBR3/FBXW7 $7^{+}+\mathrm{MTDH}$ cells compared with control cells, respectively. The inhibitory rates of proliferation of MDA-MB-231/FBXW $7^{+}$cells and SKBR3/FBXW7 ${ }^{+}$cells were reversed by $\mathrm{MTDH}\left({ }^{*} \mathrm{p}<0.05\right.$, ${ }^{* *} \mathbf{p}<0.01$ vs control, $\left.\mathbf{n}=3\right)$. C) and D) Human breast cancer cells MDA-MB-231 and SKBR3 transfected with the empty vectors or with the plasmid expressing FBXW7, or co-transfected with the plasmids expressing FBXW7 and MTDH. Cell apoptosis was detected using the Annexin V-FITC apoptosis detection kit with a flow cytometer. The increased rate of apoptosis in MDA-MB-231/FBXW $7^{+}$and MDA-MB-231/FBXW $7^{+}+\mathrm{MTDH}$ cells was 15.38 \pm 1.63 times and $8.89 \pm 0.89$ times compared with control cells. The increased rate of apoptosis in SKBR3/FBXW7 $7^{+}$and SKBR3/FBXW7 $7^{+}+M T D H$ cells was $6.29 \pm 0.96$ times and 3.20 \pm 0.54 times compared with control cells. And the apoptosis of MDA-MB-231/FBXW7 ${ }^{+}$and SKBR3/FBXW $7^{+}$was reversed by MTDH, respectively $\left({ }^{*} \mathrm{p}<0.05,{ }^{* *} \mathrm{p}<0.01\right.$ vs control, $\left.\mathrm{n}=3\right)$.

ally, FBXW7 suppresses breast cancer cell proliferation and promotes cell apoptosis by targeting MTDH for degradation. These data indicate that FBXW7 is a negative regulator of MTDH stability and functions through down-regulation of the abundance of MTDH protein level in breast cancer cells.

MTDH, firstly cloned in 2002 by subtraction hybridization [1], has been shown to play an important role in the process of cancer development and progression, including proliferation, evasion of apoptosis, cell survival, migration, and chemoresistance [30]. Recent studies show that MTDH is widely overexpressed in the majority of human malignancies. The regulation of MTDH overexpression is very complicated, and the previous research about its overexpression has been focused mainly on the transcriptional regulation [31-33]. However, in this study, we have revealed the posttranslational modifications of MTDH. The tumor suppressor FBXW7 is identified as a novel regulator of MTDH. Overexpression of any FBXW7 isoforms decreases the MTDH protein level. Importantly, knock-down of endogenous FBXW7 in HEK293 cells increases the endogenous MTDH protein level. In MDA-MB-231 cells, FBXW7a seems to be 
the major functional isoform for MTDH on endogenous level. FBXW7a interacted with MTDH and promoted its ubiquitination in vitro and in vivo, while overexpression of FBXW7 reduced the half-life of endogenous MTDH. These results support the hypothesis that FBXW7 acts as a negative regulator of MTDH and plays a critical role in regulating the level of MTDH.

In addition, it was reported that MTDH played various roles in the regulation of cancer development and the inhibition of MTDH expression could lead to cell apoptosis and proliferation arrest. Our studies suggest that FBXW7 could inhibit breast cancer cells progression through degradation of MTDH. FBXW7 was reported to be a tumor suppressor controlling the level of several oncoproteins, such as MYC, Cyclin E, Notch, KLF-5. In this study, we showed that FBXW7 inhibits MTDH accumulation. Importantly, MTDH seemed to be a crucial substrate for FBXW7 to suppress breast cancer cell proliferation. Overexpression of MTDH could reverse the FBXW7-induced breast cancer cell proliferation inhibition and cell apoptosis. Thus, FBXW7 suppresses breast cancer cell progression in some measure by controlling MTDH accumulation.

We also showed that MTDH was subject to regulation via the ubiquitin proteasome pathway by interaction with the E3 ubiquitin ligase of FBXW7. The interaction between substrate protein and E3 ubiquitin ligase has recently been emphasized in research of the overexpression of oncoproteins, and it may play an important role in retaining cancer cell progression. FBXW7 is inactivated by mutation or decreased expression in various types of human malignances [34]. Previous studies also found that FBXW7 expression level was significantly reduced in breast cancer compared to the normal breast tissues [35]. Furthermore, we propose that FBXW7 could represent a new pathway for the regulation of cell proliferation and apoptosis in breast cancer cells through negative regulating of MTDH level.

In conclusion, our studies demonstrate that FBXW7 could inhibit MTDH accumulation by targeting it for degradation through ubiquitin proteasome pathway. The turnover of MTDH by FBXW7 led to proliferation arrest and apoptosis in breast cancer cells. Given the frequent inactivation of FBXW7 in breast cancer and other cancers, these findings may help us further understand the roles of FBXW7 and MTDH in cancer development. These findings confirm that the interaction between FBXW7 and MTDH will propose a promising strategy for clinical development in tumor treatment.

Supplementary information is available in the online version of the paper.

Acknowledgments: Thanks to everyone from the Department of Clinical Laboratory for their sincere help and excellent technical assistance. This study was supported by a grant from the National Natural Science Foundation of China (No. 81001195).

\section{References}

[1] HU G, CHONG RA, YANG Q, WEI Y, BLANCO MA et al. MTDH activation by $8 \mathrm{q} 22$ genomic gain promotes chemoresistance and metastasis of poor-prognosis breast cancer. Cancer Cell 2009; 15: 9-20. doi: 10.1016/j.ccr.2008.11.013

[2] KIKUNO N, SHIINA H, URAKAMI S, KAWAMOTO K, HIRATA $\mathrm{H}$ et al. Knockdown of astrocyte-elevated gene-1 inhibits prostate cancer progression through upregulation of FOXO3a activity. Oncogene2007; 26: 7647-7655.

[3] CHEN W, KEZ, SHI H, YANG S, WANG L. Overexpression of AEG-1 in renal cell carcinoma and its correlation with tumor nuclear grade and progression. Neoplasma 2010; 57:522-529.

[4] SONG H, LI C, LI R, GENG J. Prognostic significance of AEG-1 expression in colorectal carcinoma. Int J Colorectal Dis 2010; 25: 1201-1209. doi: 10.1007/s00384-010-1009-3

[5] ZHAO Y, KONG X, LI X, YAN S, YUAN C et al. Metadherin mediates lipopolysaccharide-induced migration and invasion of breast cancer cells. PLoS One 2011; 6: e29363. doi: 10.1371/journal.pone.0029363

[6] SU P, ZHANG Q, YANG Q. Immunohistochemical analysis of Metadherin in proliferative and cancerous breast tissue. Diagn Pathol2010; 5: 38. doi: 10.1186/1746-1596-5-38

[7] LI J, ZHANG N, SONG LB, LIAO WT, JIANG LL et al. Astrocyte elevated gene-1 is a novel prognostic marker for breast cancer progression and overall patient survival. Clin Cancer Res 2008; 14: 3319-3326. doi: 10.1158/1078-0432. CCR-07-4054

[8] KANG DC, SU ZZ, SARKAR D, EMDAD L, VOLSKY DJ et al. Cloning and characterization of HIV-1-inducible astrocyte elevated gene-1, AEG-1. Gene 2005; 353: 8-15.

[9] LIU Y, KONG X, LI X, LI B, YANG Q. Knockdown of metadherin inhibits angiogenesis in breast cancer. Int J Oncol 2015; 46: 2459-2466. doi: 10.3892/ijo.2015.2973

[10] WANG J, MALDONADO MA. The ubiquitin-proteasome system and its role in inflammatory and autoimmune diseases. Cell Mol Immunol 2006; 3: 255-261.

[11] TEIXEIRA LK, REED SI. Ubiquitin ligases and cell cycle control. Annu Rev Biochem 2016; 82: 387-414. doi: 10.1146/ annurev-biochem-060410-105307

[12] CAO J, GE MH, LING ZQ. Fbxw7 Tumor Suppressor: A Vital Regulator Contributes to Human Tumorigenesis. Medicine (Baltimore) 2016; 95: e2496. doi: 10.1097/ MD.0000000000002496

[13] RAJAGOPALAN H, JALLEPALLI PV, RAGO C, VELCULESCU VE, KINZLER KW et al. Inactivation of hCDC4 can cause chromosomal instability. Nature 2004; 428: 77-81.

[14] HAGEDORN M, DELUGIN M, ABRALDES I, ALLAIN N, BELAUD-ROTUREAU MA et al. FBXW7/hCDC4 controls glioma cell proliferation in vitro and is a prognostic marker for survival in glioblastoma patients. Cell Div 2007; 2: 9.

[15] YE X, NALEPA G, WELCKER M, KESSLER BM, SPOONER E et al. Recognition of phosphodegron motifs in human cyclin E by the SCF(Fbw7) ubiquitin ligase. J Biol Chem 2004; 279: 50110-50119.

[16] WELCKER M, ORIAN A, JIN J, GRIM JE, HARPER JW et al. The Fbw7 tumor suppressor regulates glycogen synthase kinase 3 phosphorylation-dependent c-Myc protein degradation. Proc Natl Acad Sci U S A 2004; 101: 9085-9090. 
[17] YADA M, HATAKEYAMA S, KAMURA T, NISHIYAMA $\mathrm{M}$, TSUNEMATSU $\mathrm{R}$ et al. Phosphorylation-dependent degradation of c-Myc is mediated by the F-box protein Fbw7. EMBO J 2004; 23: 2116-2125.

[18] KOEPP DM, SCHAEFER LK, YE X, KEYOMARSI K, CHU $\mathrm{C}$ et al. Phosphorylation-dependent ubiquitination of cyclin $\mathrm{E}$ by the SCFFbw7 ubiquitin ligase. Science 2001; 294: 173-177.

[19] TETZLAFF MT, YU W, LI M, ZHANG P, FINEGOLD M et al. Defective cardiovascular development and elevated cyclin $\mathrm{E}$ and Notch proteins in mice lacking the Fbw7 F-box protein. Proc Natl Acad Sci U S A 2004; 101: 3338-3345.

[20] LIU N, LI H, LI S, SHEN M, XIAO N et al. The Fbw7/human CDC4 tumor suppressor targets proproliferative factor KLF5 for ubiquitination and degradation through multiple phosphodegron motifs. J Biol Chem 2010; 285: 18858-18867. doi: 10.1074/jbc.M109.099440

[21] LOCHAB S, PAL P, KAPOOR I, KANAUJIYA JK, SANYAL $\mathrm{S}$ et al. E3 ubiquitin ligase Fbw7 negatively regulates granulocytic differentiation by targeting G-CSFR for degradation. Biochim Biophys Acta 2013; 1833: 2639-2652. doi: 10.1016/j. bbamcr.2013.06.018

[22] DAVIS RJ, WELCKER M, CLURMAN BE. Tumor suppression by the Fbw7 ubiquitin ligase: mechanisms and opportunities. Cancer Cell 2014; 26: 455-464. doi: 10.1016/j. ccell.2014.09.013

[23] BISWAS M, PHAN D, WATANABE M, CHAN JY. The Fbw7 tumor suppressor regulates nuclear factor E2-related factor 1 transcription factor turnover through proteasomemediated proteolysis. J Biol Chem 2011; 286: 39282-39289. doi: 10.1074/jbc.M111.253807

[24] CHEN X, SHEN J, LI X, WANG X, LONG M et al. Rlim, an E3 ubiquitin ligase, influences the stability of Stathmin protein in human osteosarcoma cells. Cell Signal 2014; 26: 1532-1538. doi: 10.1016/j.cellsig.2014.03.018

[25] GAO ZW, WANG HP, DONG K, LIN F, WANG X et al. Adenosine inhibits migration, invasion and induces apoptosis of human cervical cancer cells. Neoplasma 2016; 63: 201207. doi: 10.4149/204_150723N407
[26] FUJII Y, YADA M, NISHIYAMA M, KAMURA T, TAKAHASHI $\mathrm{H}$ et al. Fbxw7 contributes to tumor suppression by targeting multiple proteins for ubiquitin-dependent degradation. Cancer Sci 2006; 97: 729-736.

[27] GRIM JE, GUSTAFSON MP, HIRATA RK, HAGAR AC, SWANGER J et al. Isoform- and cell cycle-dependent substrate degradation by the Fbw7 ubiquitin ligase. J Cell Biol 2008; 181: 913-920. doi: 10.1083/jcb.200802076

[28] STROHMAIER H, SPRUCK CH, KAISER P, WON KA, SANGFELT O et al. Human F-box protein hCdc4 targets cyclin $\mathrm{E}$ for proteolysis and is mutated in a breast cancer cell line. Nature 2001; 413: 316-322.

[29] XU W, TARANETS L, POPOV N. Regulating Fbw7 on the road to cancer. Semin Cancer Biol 2016; 36: 62-70. doi: 10.1016/j.semcancer.2015.09.005

[30] SU ZZ, KANG DC, CHEN Y, PEKARSKAYA O, CHAO W et al. Identification and cloning of human astrocyte genes displaying elevated expression after infection with HIV-1 or exposure to HIV-1 envelope glycoprotein by rapid subtraction hybridization, RaSH. Oncogene 2002; 21: 3592-3602.

[31] BRITT DE, YANG DF, YANG DQ, FLANAGAN D, CALLANAN $\mathrm{H}$ et al. Identification of a novel protein, LYRIC, localized to tight junctions of polarized epithelial cells. Exp Cell Res 2004; 300: 134-148.

[32] LEE SG, SU ZZ, EMDAD L, SARKAR D, FISHER PB. Astrocyte elevated gene-1 (AEG-1) is a target gene of oncogenic Ha-ras requiring phosphatidylinositol 3-kinase and c-Myc. Proc Natl Acad Sci U S A 2006; 103: 17390-17395.

[33] NOCH E, BOOKLAND M, KHALILI K. Astrocyte-elevated gene-1 (AEG-1) induction by hypoxia and glucose deprivation in glioblastoma. Cancer Biol Ther 2011; 11: 32-39.

[34] AKHOONDI S, SUN D, VON DER LEHR N, APOSTOLIDOU S, KLOTZ K et al. FBXW7/hCDC4 is a general tumor suppressor in human cancer. Cancer Res 2007; 67: 9006-9012.

[35] WEI G, WANG Y, ZHANG P, LU J, MAO JH. Evaluating the prognostic significance of FBXW7 expression level in human breast cancer by a meta-analysis of transcriptional profiles. J Cancer Sci Ther 2012; 4: 299-305. 